\title{
Disk or halo white dwarfs?
}

\section{Kinematic analysis of high proper motion surveys}

\author{
A. Spagna, D. Carollo, M. G. Lattanzi, and B. Bucciarelli
}

INAF - Osservatorio Astronomico di Torino, 10025 Pino Torinese, Italy

e-mail: spagna@to.astro.it

Received 13 April 2004 / Accepted 21 July 2004

\begin{abstract}
We present an alternative method for the kinematic analysis of high proper motion surveys and discuss its application to the survey of Oppenheimer et al. (2001) for the selection of reliable halo white dwarfs (WDs). The local WD space density we estimate is $\rho_{\mathrm{WD}} \simeq 1 \div 2 \times 10^{-5} M_{\odot} \mathrm{pc}^{-3}$, which is about an order of magnitude smaller than the value derived in Oppenheimer et al. (2001), and is consistent with the values obtained from recent reanalyses of the same data. Our result, which corresponds to a fraction of $0.1 \% \div 0.2 \%$ of the local dark matter, does not support the scenario suggested by microlensing experiments that ancient cool WDs could contribute significantly to the dark halo of the Milky Way.
\end{abstract}

Key words. stars: kinematics - white dwarfs - dark matter - Galaxy: stellar content - surveys - methods: statistical

\section{Introduction}

One of the most recent challenges in observational astronomy is to explain the nature of the objects that produced the microlensing events towards the Magellanic Clouds (Alcock et al. 2000). The most obvious candidates for these events are ancient white dwarfs, and several projects have been carried out in recent years to reveal the existence of such a hidden population of dim sources (see Hansen \& Liebert 2003 for a review). The most extensive survey to date is that of Oppenheimer et al. (2001, OHDHS). They discovered 38 suspected halo white dwarfs and derived a local density of $\sim 1.1 \times 10^{-4} M_{\odot} \mathrm{pc}^{-3}$, which corresponds to a fraction of $1-2 \%$ of the halo dark matter in the vicinity of the Sun. Different authors challenged these results on the basis of the age estimates of the candidates (Hansen 2001; Bergeron 2003), or after a reanalysis of the kinematic data (e.g. Reid et al. 2001; Reylé et al. 2001; Flynn et al. 2003; Torres et al. 2002).

These studies reveal a significant contamination of thick disk objects affecting the halo WD sample, and point out the basic problem of defining an accurate procedure to deconvolve the halo and thick disk populations on the basis of their kinematic and photometric properties.

In this paper we describe a general statistical method designed to reject objects with disk kinematics and isolate probable halo members from the screening of kinematically selected samples. We discuss the results obtained with this method when applied to the OHDHS survey, and compare them to the preliminary results derived from the GSC II-based new high proper motion survey in the Northern hemisphere by Carollo et al. (2004).

\section{SSS Halo WD survey}

The OHDHS survey was based on digitized, photographic Schmidt plates (R59F and $B_{J}$ passbands) from the SuperCOSMOS Sky Survey (SSS, Hambly et al. 2001). They analyzed 196 three epoch plates (IIIaJ, IIIaF and IV-N) covering an area of 4165 square degrees near the South Galactic Pole (SGP). The magnitude limit of the survey is of $R 59 F=19.8$, while the proper motion limits are $0.33^{\prime \prime} \mathrm{yr}^{-1}<$ $\mu<10^{\prime \prime} \mathrm{yr}^{-1}$. They found 98 WDs, whose tangential velocities were derived from the measured proper motions and photometric distances estimated via a linear color magnitude (CM) relation, $M_{B_{J}}$ vs. $B_{J}-R 59 F$, calibrated by means of the WD sample with available trigonometric parallaxes published by Bergeron et al. (1997). The kinematic analysis of this sample was made in the two dimensional $(U, V)$ plane, after assuming that the third galactic velocity component was zero $(W=0)$. Thick disk contaminants were rejected with a $2 \sigma$ threshold, $\sqrt{U^{2}+(V+35)^{2}}>95 \mathrm{~km} \mathrm{~s}^{-1}$, which would correspond to a $86 \%$ confidence level in the case of a nonkinematically selected sample. In this way, 38 WDs were considered as halo members, from which a space density of $\rho_{\mathrm{WD}} \simeq$ $1.1 \times 10^{-4} M_{\odot} \mathrm{pc}^{-3}$ was computed, assuming $0.6 M_{\odot}$ for the average WD mass.

As mentioned in the previous section, these results were critically revised by several authors. In particular, an independent kinematic analysis of the OHDHS sample was performed by Reid et al. (2001), who noted that the resulting distribution of the WDs in the $(U, V)$ diagram seems more compatible with the high velocity tails of the thick disk. They computed $(U, V)$ components assuming that the unknown radial velocity 


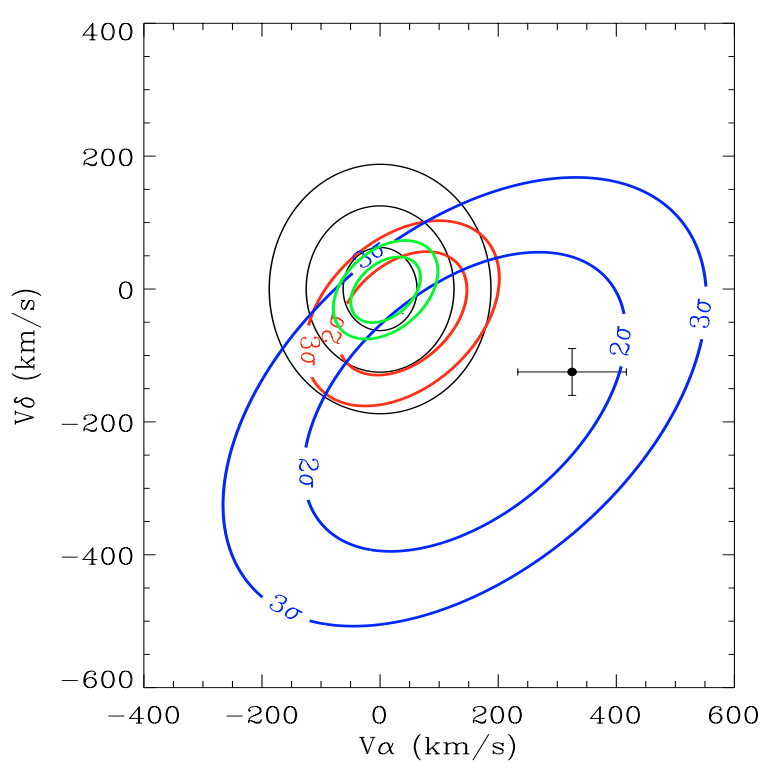

Fig. 1. Tangential velocity distributions, $\psi\left(V_{\alpha}, V_{\delta}\right)$, toward the direction $\left(l=143^{\circ}, b=-57^{\circ}\right)$ of WD0135-039 (solid circle). The ellipses show the iso-probability contours $(2 \sigma, 3 \sigma)$ of the thin disk, thick disk, and local halo populations based on the kinematic parameters from Binney \& Merrifield (1998), Soubiran et al. (2003) and Casertano et al. (1990), respectively. The three concentric circles indicate the velocity thresholds, $V_{\min }=4.74 \mu_{\lim } r$, for the OHDHS survey $\left(\mu_{\lim }=0.33^{\prime \prime} \mathrm{yr}^{-1}\right)$ at the distances $r=40 \mathrm{pc}, 80 \mathrm{pc}$ and $120 \mathrm{pc}$.

is null $\left(V_{r}=0\right)$ and selected halo WDs with the crude but robust criterion of accepting objects with retrograde motion only (4 objects). This leads to a more conservative value of the density, $\rho_{\mathrm{WD}} \simeq 1.8 \times 10^{-5} M_{\odot} \mathrm{pc}^{-3}$.

Recently, Salim et al. (2004) reanalyzed the WD sample of OHDHS on the basis of new spectroscopic and photometric measurements. Radial velocities of 13 WDs with $\mathrm{H}_{\alpha}$ lines, and standard Johnson-Cousins photometry for half of the sample were obtained. In addition, distances were redetermined by CCD photometry and adopting the theoretical color magnitude relation for hydrogen and helium atmospheres published by Bergeron, Leggett \& Ruiz (2001). Salim et al. (2004) confirmed the results of OHDHS with the same $95 \mathrm{~km} \mathrm{~s}^{-1}(2 \sigma)$ threshold, but showed that a minimum density, $n_{\mathrm{WD}} \simeq 3.1 \times$ $10^{-5} \mathrm{pc}^{-3}$ is attained with a higher, more conservative, threshold of $190 \mathrm{~km} \mathrm{~s}^{-1}$.

\section{Kinematic analysis}

The kinematic analysis of the WD sample drawn from a proper motion limited survey, including the choice of a criterium for rejecting the contaminant disk WDs and select the true halo WDs, is one of the critical steps of this kind of study.

As the velocity distribution of the $\operatorname{disk}(\mathrm{s})$ and halo population do partially overlap (Fig. 1), it is not possible to infer unequivocally, on the basis of kinematic data alone, the parent population of each object. Nevertheless, it is always possible to test if an object is, or is not, consistent with the velocity distribution of a certain population once a value for the confidence level is chosen.
Here, we retain as halo WDs those objects whose kinematics are not consistent with the velocity distribution of the thick disk population ${ }^{1}$ given a certain confidence level; this allows the identification of halo WDs while limiting the contamination by high velocity thick disk objects. Unless corrected for the incompleteness due to the fraction of rejected halo WDs whose kinematics are compatible with that of the thick disk population, it is clear that this procedure can only provide a lower limit to the actual density.

An alternative, and potentially more rigorous procedure, is a Maximum-likelihood analysis that fits simultaneously the superposition of two or more populations (see e.g. Nelson et al. 2002; Koopmans \& Blandford 2001). In this case however, because of the small size of the samples, further assumptions on the kinematics and the formation process (IMF, age, etc.) of all the populations involved are usually necessary.

\subsection{Schwarzschild distribution}

We assume that the probability that the galactic velocity components $(U, V, W)$ of an object in the solar neighborhood belonging to a certain stellar population lying in the element of velocity space $\mathrm{d}^{3} \bar{v}=\mathrm{d} U \mathrm{~d} V \mathrm{~d} W$ is well described by a Schwarzschild distribution:

$p(\bar{v})=\frac{1}{(2 \pi)^{3 / 2} \sigma_{U} \sigma_{V} \sigma_{W}} \exp \left[-\frac{U^{2}}{2 \sigma_{U}^{2}}-\frac{\left(V-V_{0}\right)^{2}}{2 \sigma_{V}^{2}}-\frac{W^{2}}{2 \sigma_{W}^{2}}\right]$

which represents a trivariate Gaussian ellipsoid, where $V_{0}$ indicates the rotation lag with respect to the LSR and $\sigma_{U}, \sigma_{V}$, and $\sigma_{W}$ the velocity dispersions.

In practice, the galactic components need to be derived from the observed tangential and radial velocity components $\left(V_{\alpha}, V_{\delta}, V_{r}\right)$ :

$$
\left[\begin{array}{c}
U \\
V \\
W
\end{array}\right]=\boldsymbol{G}_{2000}\left[\begin{array}{c}
V_{\alpha} \\
V_{\delta} \\
V_{r}
\end{array}\right]+\left[\begin{array}{c}
U_{\odot} \\
V_{\odot} \\
W_{\odot}
\end{array}\right]
$$

where $\boldsymbol{G}_{2000}=\boldsymbol{G}(\alpha, \delta)$ is the transformation matrix from the equatorial coordinates system (J2000) to the galactic system, which depends explicitly on the stellar position $(\alpha, \delta)$. Here, $\left(U_{\odot}, V_{\odot}, W_{\odot}\right)$ is the Sun velocity with respect to the Local Standard of Rest (LSR), for which Dehnen \& Binney (1998) estimated $(+10.00 \pm 0.36,+5.25 \pm 0.62,+7.17 \pm 0.38) \mathrm{km} \mathrm{s}^{-1}$ from the analysis of the Hipparcos catalogue. The tangential velocities $V_{\alpha}$ and $V_{\delta}\left(\mathrm{km} \mathrm{s}^{-1}\right)$ are computed from the observed proper motions $\left(\operatorname{arcsec} \mathrm{yr}^{-1}\right)$ and distances $(\mathrm{pc})$ derived from trigonometric or photometric parallaxes, $\pi=1 / d$, as usual:

$V_{\alpha}=4.74047 d \mu_{\alpha} \cos \delta$

$V_{\delta}=4.74047 d \mu_{\delta}$.

\subsection{Tangential velocity distribution}

If the full $3 \mathrm{D}$ space velocity cannot be recovered, as in the case of proper motion surveys, we can adopt a similar procedure in the $2 \mathrm{D}$ tangential velocity plane, $\left(V_{\alpha}, V_{\delta}\right)$. The bivariate

\footnotetext{
${ }^{1}$ Implicitly, we assume that besides the thick disk WDs, this criterion rejects the "slowest" thin disk objects as well.
} 
marginal distribution, $\psi\left(V_{\alpha}, V_{\delta}\right)$, can be obtained by properly integrating the distribution in Eq. (1) along the $V_{r}$ component:

$$
\begin{aligned}
\psi\left(V_{\alpha}, V_{\delta}\right)= & \frac{1}{2 \pi \sigma_{\alpha} \sigma_{\delta} \sqrt{1-\rho^{2}}} \\
& \times \exp \left[\frac { - 1 } { 2 ( 1 - \rho ^ { 2 } ) } \left(\frac{\left(V_{\alpha}-V_{\alpha 0}\right)^{2}}{\sigma_{V \alpha}^{2}}\right.\right. \\
& \left.\left.-2 \rho \frac{\left(V_{\alpha}-V_{\alpha 0}\right)}{\sigma_{V \alpha}} \frac{\left(V_{\delta}-V_{\delta 0}\right)}{\sigma_{V \delta}}+\frac{\left(V_{\delta}-V_{\delta 0}\right)^{2}}{\sigma_{V \delta}^{2}}\right)\right] .
\end{aligned}
$$

This is a general bivariate Gaussian distribution which is defined by five parameters: $V_{\alpha 0}, V_{\delta 0}, \sigma_{V \alpha}, \sigma_{V \delta}$ and $\rho$. These parameters are linear functions of the first and second order moments of Eq. (1), as described for instance in Trumpler \& Weaver (1953).

Our analysis will be based on Eq. (3), that represents the appropriate density distribution when radial velocities are missing. Notice that this approach, even in the case of surveys involving widely different line-of-sights, allows the derivation of the exact tangential velocity distribution for each star, without any assumption on the unknown third velocity component $V_{r}$.

\subsection{Thick disk model}

The following properties for the population of thick disk WDs in the solar neighborhood were assumed:

- a uniform local space density; the typical distance reachable by ground based surveys $(\sim 100 \mathrm{pc})$ is much smaller than the exponential vertical scale-height of the thick disk $\left(h_{z} \simeq 1000 \mathrm{pc}\right)$;

- a velocity distribution (Eq. (1)) with $\left(\sigma_{U}, \sigma_{V}, \sigma_{W}, V_{0}\right) \simeq$ $(63,39,39,-45) \mathrm{km} \mathrm{s}^{-1}$, as derived in Soubiran, Bienaymè \& Siebert (2003).

We notice that the velocity ellipsoid of the thick disk population is not currently well established so that this choice will affect the final result. For instance, the presence of a non-Gaussian high velocity tail (cf. Gilmore et al. 2002) would increase the contamination affecting the halo WD sample.

\subsection{Kinematically selected samples}

In the case of a magnitude- and $\mu$-limited survey with a total extension of $\Omega$ steradians, the following observational constraints need to be taken into account:

1. an apparent magnitude limit $m<m_{\text {lim }}$ which implies a distance limit as a function of the absolute magnitude, $M$, of the target:

$r<r_{\max }(M)=10^{\left[0.2\left(m_{\lim }-M\right)+1\right]}$

which, in turn, defines the maximum volume ${ }^{2}, V_{\mathrm{Max}}(M)=$ $\frac{1}{3} \Omega r_{\max }^{3}$ covered by the survey;

${ }^{2}$ Note that this is a purely photometric definition which does not correspond exactly to the analogue quantity adopted for the evaluation of the WD density via the $1 / V_{\max }$ method (Schmidt 1975).
2. a proper motion limit $\mu>\mu_{\text {lim }}$ which translates into a tangential velocity threshold varying with stellar distance:

$$
V_{\tan }=\sqrt{V_{\alpha}^{2}+V_{\delta}^{2}}>V_{\min }(r)=4.74047 \mu_{\lim } r .
$$

Note that, although the distance distribution $\left(\propto r^{2}\right)$ and the kinematic distribution (Eq. (3)) of the complete population are independent, now they are correlated for the observed sample because of the existence of the velocity threshold, $V_{\min }(r)$.

The probability of selecting a star with absolute magnitude $M$ in the range $(r, r+\mathrm{d} r),\left(V_{\alpha}, V_{\alpha}+\mathrm{d} V_{\alpha}\right),\left(V_{\delta}, V_{\delta}+\mathrm{d} V_{\delta}\right)$ is then $\mathrm{d} P=f\left(r, V_{\alpha}, V_{\delta}\right) \mathrm{d} r \mathrm{~d} V_{\alpha} \mathrm{d} V_{\delta}$, where the joint probability density is:

$f\left(r, V_{\alpha}, V_{\delta}\right)= \begin{cases}K r^{2} \psi\left(V_{\alpha}, V_{\delta}\right) & \text { if } V_{\tan }>V_{\min }(r) \\ & \text { and } r<r_{\max }(M) \\ 0 & \text { if } V_{\tan } \leq V_{\min }(r) \\ & \text { or } r \geq r_{\max }(M) .\end{cases}$

Here, $K$ is a normalization constant such that $\iiint f \mathrm{~d} r \mathrm{~d} V_{\alpha} \mathrm{d} V_{\delta}=1$.

If we integrate over $r$ the joint probability density function given in Eq. (4), we obtain the marginal density distribution

$h\left(V_{\alpha}, V_{\delta}\right)=\int_{0}^{r_{\max }} f\left(r, V_{\alpha}, V_{\delta}\right) \mathrm{d} r$

which quantifies the probability that an object with tangential velocities $\left(V_{\alpha}, V_{\delta}\right)$ could be randomly found somewhere within the whole volume $\frac{1}{3} \Omega r_{\text {max }}^{3}$, where an object with absolute magnitude $M$ could in principle be observed.

At the same time, we can introduce the (conditional) probability that an object with tangential velocities $\left(V_{\alpha}, V_{\delta}\right)$ is found at the measured distance, $r$ :

$t\left(V_{\alpha}, V_{\delta} \mid r\right)=\frac{f\left(r, V_{\alpha}, V_{\delta}\right)}{g(r)}$

where

$g(r)=\iint_{-\infty}^{+\infty} f\left(r, V_{\alpha}, V_{\delta}\right) \mathrm{d} V_{\alpha} \mathrm{d} V_{\delta}$

is the marginal density distribution which defines the probability that an object with any velocity can be observed at a distance $r$. Because the velocity threshold increases linearly with distance, $V_{\min } \propto r$, the space distribution of the proper-motion selected sample (Eq. (7)) is also biased towards smaller distances.

Both the marginal distribution $h\left(V_{\alpha}, V_{\delta}\right)$ and the conditional probability $t\left(V_{\alpha}, V_{\delta} \mid r\right)$ can be used to test the consistency of each object with a parent population. In principle, the conditional probability $t\left(V_{\alpha}, V_{\delta} \mid r\right)$ seems more appropriate than $h\left(V_{\alpha}, V_{\delta}\right)$ since it fully utilizes the individual stellar distances. However, the differences become insignificant when the confidence level is set to sufficiently high values (see next section).

Note that, formally, Eq. (6) is equivalent to the original distribution, $\psi\left(V_{\alpha}, V_{\delta}\right)$, except that the probability is null for $\sqrt{V_{\alpha}^{2}+V_{\delta}^{2}} \leq V_{\min }(r)$, and it has been re-normalized. 

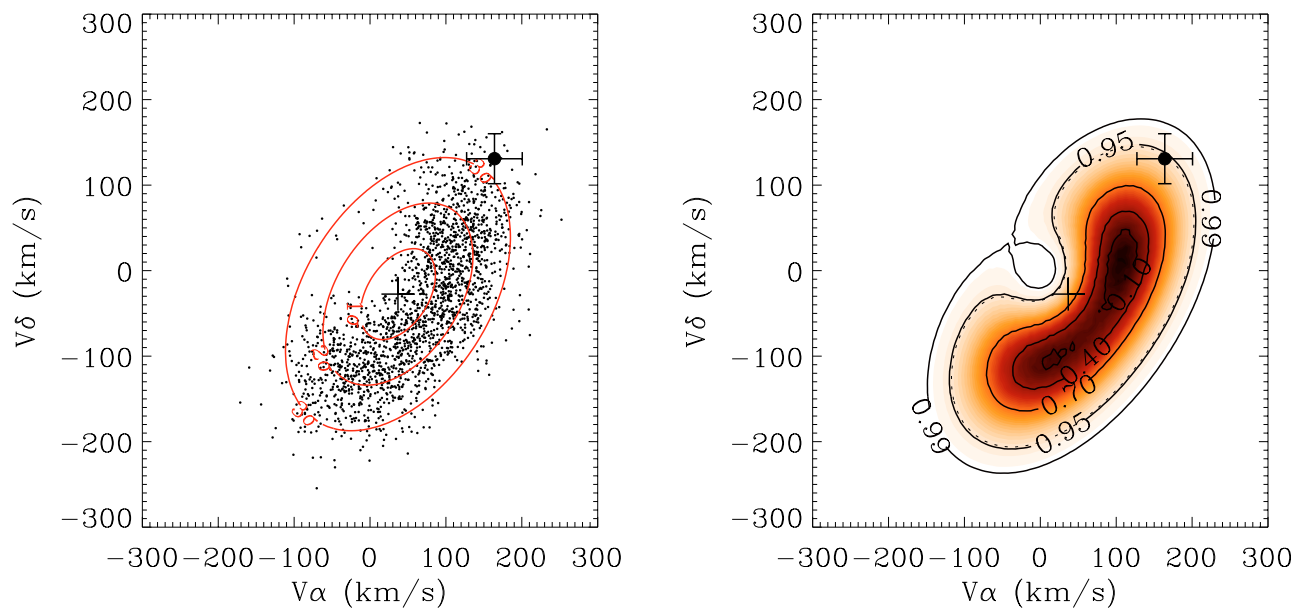

Fig. 2. Left panel: iso-probability contours $(1 \sigma, 2 \sigma, 3 \sigma)$ of $\psi\left(V_{\alpha}, V_{\delta}\right)$ compared to a Montecarlo simulation (dots) of the thick disk stars in the direction $\left(l=226^{\circ} 34, b=-64^{\circ} .27\right)$ of LHS 1447 (solid circle) drawn from the kinematically selected distribution, $h\left(V_{\alpha}, V_{\delta}\right)$. Right panel: iso-probability contours (confidence levels of $40 \%, 70 \%, 95 \%$ and $99 \%)$ of $h\left(V_{\alpha}, V_{\delta}\right)$.
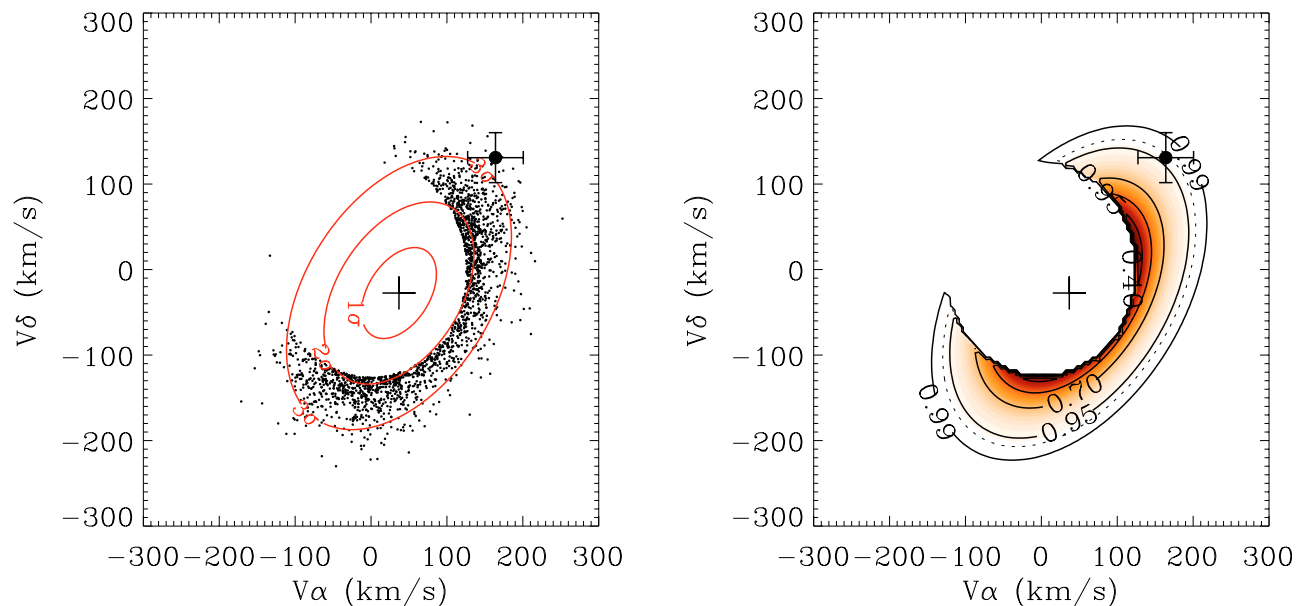

Fig. 3. Left panel: iso-probability contours $(1 \sigma, 2 \sigma, 3 \sigma)$ of $\psi\left(V_{\alpha}, V_{\delta}\right)$ compared to a Montecarlo simulation (dots) of the thick disk stars in the direction $\left(l=226.34, b=-64^{\circ} .27\right)$ of LHS 1447 (solid circle) drawn from the kinematically selected distribution, $t\left(V_{\alpha}, V_{\delta} \mid r\right)$. Right panel: iso-probability contours (confidence levels of $40 \%, 70 \%, 95 \%$ and $99 \%)$ of $t\left(V_{\alpha}, V_{\delta} \mid r\right)$.

\subsection{Confidence intervals and contamination}

Because a proper motion limited survey undersamples the low velocity objects, the main difference between the kinematically selected distributions (Eqs. (5)-(6)) and the complete one (Eq. (3)) is that the probability density is redistributed from the low velocity regions towards the high velocity tails. This means that the observed sample is biased towards high velocity objects, as shown for instance by the simulations of Reylé et al. (2001) and Torres et al. (2002).

This effect needs to be taken into account when we define a confidence interval over the $\left(V_{\alpha}, V_{\delta}\right)$ plane in order to test the consistency with the parent population and to estimate the contamination due to objects in the tails beyond the critical limit. In fact, the adoption of the original $\psi\left(V_{\alpha}, V_{\delta}\right)$ to reject the disk stars with respect to a certain confidence level, e.g. $1-\alpha=99 \%$, would exclude $99 \%$ of all the existing thick disk stars which, however, corresponds to a smaller fraction of the thick disk objects that are really present in the kinematically selected sub-sample. In this case, only the confidence interval defined for $t\left(V_{\alpha}, V_{\delta} \mid r\right)$, or $h\left(V_{\alpha}, V_{\delta}\right)$, assures that the fraction of false negatives contaminating the sample of bona fide halo stars does not exceed - on average $-1 \%$ of the observed thick disk objects.

In the left panels of Figs. 2-3 the concentric ellipses show the iso-probability contours $(1 \sigma, 2 \sigma, 3 \sigma)$ of the velocity distribution expected for thick disk stars, $\psi\left(V_{\alpha}, V_{\delta}\right)$, evaluated in the direction of one of the stars in Oppenheimer's sample (LHS 1447), whose tangential velocity is marked with a filled circle. The points represent a Montecarlo realization of 2000 simulated WDs drawn from the kinematically selected distributions $h\left(V_{\alpha}, V_{\delta}\right)$ and $t\left(V_{\alpha}, V_{\delta} \mid r\right)$. The excess of "simulated" thick disk stars with high velocity is shown by the fact that there are many more than $\sim 20$ objects ( $1 \%$ of the simulated sample) outside the $3 \sigma$ confidence interval.

LHS 1447 is also located outside the $3 \sigma$ contour so that, according to the complete distribution, it should be rejected as a thick disk star with a confidence level higher than $1-\alpha=99 \%$. Actually, that conclusion would be incorrect if we tested the hypothesis that LHS 1447 is a member of the kinematically 
Table 1. Estimation of the halo WD density based on the objects selected from the OHDHS sample.

\begin{tabular}{|c|c|c|c|c|c|c|}
\hline \multirow{2}{*}{$\begin{array}{r}\text { Confid. } \\
\text { level }\end{array}$} & \multicolumn{2}{|r|}{$\psi\left(V_{\alpha}, V_{\delta}\right)$} & \multicolumn{2}{|r|}{$h\left(V_{\alpha}, V_{\delta}\right)$} & \multicolumn{2}{|r|}{$t\left(V_{\alpha}, V_{\delta} \mid r\right)$} \\
\hline & WDs & $\rho_{\mathrm{WD}}\left(M_{\odot} \mathrm{pc}^{-3}\right)$ & WDs & $\rho_{\mathrm{WD}}\left(M_{\odot} \mathrm{pc}^{-3}\right)$ & WDs & $\rho_{\mathrm{WD}}\left(M_{\odot} \mathrm{pc}^{-3}\right)$ \\
\hline $99 \%$ & 14 & $(2.0 \pm 0.9) \times 10^{-5}$ & 10 & $(1.6 \pm 0.8) \times 10^{-5}$ & 10 & $(1.5 \pm 0.8) \times 10^{-5}$ \\
\hline $95 \%$ & 20 & $(3.1 \pm 1.0) \times 10^{-5}$ & 12 & $(1.9 \pm 0.9) \times 10^{-5}$ & 13 & $(2.0 \pm 0.9) \times 10^{-5}$ \\
\hline
\end{tabular}

Table 2. Estimation of the halo WD density based on the sample revised by Salim et al. (2004).

\begin{tabular}{|c|c|c|c|c|c|c|}
\hline \multirow{2}{*}{$\begin{array}{r}\text { Confid. } \\
\text { level }\end{array}$} & \multicolumn{2}{|r|}{$\psi\left(V_{\alpha}, V_{\delta}\right)$} & \multicolumn{2}{|r|}{$h\left(V_{\alpha}, V_{\delta}\right)$} & \multicolumn{2}{|r|}{$t\left(V_{\alpha}, V_{\delta} \mid r\right)$} \\
\hline & WDs & $\rho_{\mathrm{WD}}\left(M_{\odot} \mathrm{pc}^{-3}\right)$ & WDs & $\rho_{\mathrm{WD}}\left(M_{\odot} \mathrm{pc}^{-3}\right)$ & WDs & $\rho_{\mathrm{WD}}\left(M_{\odot} \mathrm{pc}^{-3}\right)$ \\
\hline $99 \%$ & 19 & $(1.8 \pm 0.6) \times 10^{-5}$ & 17 & $(1.6 \pm 0.6) \times 10^{-5}$ & 16 & $(1.5 \pm 0.6) \times 10^{-5}$ \\
\hline $95 \%$ & 28 & $(3.3 \pm 0.8) \times 10^{-5}$ & 18 & $(1.8 \pm 0.6) \times 10^{-5}$ & 18 & $(1.9 \pm 0.6) \times 10^{-5}$ \\
\hline
\end{tabular}

selected sample as shown in the right panels of Figs. 2-3, where the marginal and conditional distributions, $h\left(V_{\alpha}, V_{\delta}\right)$ and $t\left(V_{\alpha}, V_{\delta} \mid r\right)$, are drawn. In these cases the star is located within the iso-probability contour delimiting the $99 \%$ confidence level so that it must be accepted as a thick disk star.

\section{Results and discussion}

Both distributions, $h\left(V_{\alpha}, V_{\delta}\right)$ and $t\left(V_{\alpha}, V_{\delta} \mid r\right)$, were used to analyze the WD sample in the OHDHS survey.

The kinematic tests were carried out in the tangential plane of each individual star so that no assumption on radial velocity is necessary. The values of $95 \%$ and $99 \%$ for the confidence level $(1-\alpha)$ were chosen in order to minimize the presence of false negatives. With a total sample of 98 WDs, presumably a mixture of (thin and thick) disk and halo WDs, we expect that $<1(99 \%)$ and $<5(95 \%)$ of the high velocity thick disk stars would contaminate the selected Pop. II WD sample.

\subsection{Halo WD density}

In Table 1 we report the results based on this procedure for the WD sample published by OHDHS. We only found 10 objects which do not appear consistent with the kinematically selected density distributions, $h\left(V_{\alpha}, V_{\delta}\right)$ and $t\left(V_{\alpha}, V_{\delta} \mid r\right)$, at the $99 \%$ confidence level, while 12-13 probable halo WDs are selected when $1-\alpha=95 \%$. As expected, the number of candidates increases up to $14(99 \%)$ or $20(95 \%)$ in the case of a test based on the complete distribution, $\psi\left(V_{\alpha}, V_{\delta}\right)$, mainly because of a higher contamination.

Finally, the halo WD density was estimated by means of the classical $1 / \mathrm{V}_{\text {Max }}$ method (Schmidt 1975), and assuming a value of $0.6 M_{\odot}$ for the typical WD mass. The results, with their (Poissonian only) errors, are reported in Table 1, where the different values refer to the two confidence levels and the three probability distributions used for the calculations.

Although affected by large uncertainties, the values in Table 1 suggest a density of $\rho_{\mathrm{WD}} \approx 10^{-5} M_{\odot} \mathrm{pc}^{-3}$, i.e. $0.1-0.2 \%$ of the local dark matter, which is an order of magnitude smaller than that reported in OHDHS.
Our results are consistent with the local mass density of halo WDs estimated by Gould et al. (1998), and with various reanalyses of the OHDHS sample (e.g. Reid et al. 2001; Reylé et al. 2001; Torres et al. 2002; Salim et al. 2004). Furthermore, Carollo et al. (2004), applying the statistical methodology described in this paper to a new high proper motion survey based on GSC-II material, derived a similar value of $\sim 10^{-5} M_{\odot} \mathrm{pc}^{-3}$.

Lacking individual trigonometric parallaxes, a critical point of this (and any) analysis is the choice of the method for the estimation of the distances, which directly affects the evaluation of the WD tangential velocities and, of course, of their stellar density. As remarked by several authors (see e.g. Torres et al. 2002; Bergeron 2003), empirical and theoretical CM relations can both give rise to systematic errors.

If for the distances of the OHDHS sample we adopt the values recently redetermined ${ }^{3}$ by Salim et al. (2004), the number of selected halo WDs increases but the resulting densities, shown in Table 2, are not significantly different from those reported in Table 1.

\subsection{Distance and velocity errors}

The large error, $\sim 20-30 \%$, affecting WD photometric parallaxes, cannot be neglected in a rigorous statistical analysis. Besides the contribution of the photometric errors, the large uncertainty in the distance modulus, $m-M$, derives from the large intrinsic dispersion ( $\sigma_{\mathrm{Mv}} \simeq 0.4-0.5 \mathrm{mag}$ ) of the CM relation, a consequence of the superposition of cooling sequences of WDs of different masses and atmospheres.

In practice, the main effect of the tangential velocity errors, $\epsilon_{V} / V=\sqrt{\left(\sigma_{\mu} / \mu\right)^{2}+\left(\sigma_{d} / d\right)^{2}}$, is to increase the dispersion and the overlap of the "observed" kinematic distributions belonging to the various stellar populations. Clearly this also increases the contamination of the disk WDs and makes the identification of the halo WDs more difficult.

Although a more rigorous statistical analysis would be necessary to properly consider the presence of these errors, a

3 They adopted CM relations based on theoretical cooling tracks of $0.6 M_{\odot}$ WDs with $\mathrm{H}$ or $\mathrm{He}$ atmospheres. This resulted in distances systematically $16 \%$ larger (on average) than those in OHDHS. 
Table 3. Same as Table 1 after adopting a thick disk velocity distribution convolved with the observation errors.

\begin{tabular}{|c|c|c|c|c|c|c|}
\hline \multirow{2}{*}{$\begin{array}{r}\text { Confid. } \\
\text { level }\end{array}$} & \multicolumn{2}{|r|}{$\psi\left(V_{\alpha}, V_{\delta}\right)$} & \multicolumn{2}{|r|}{$h\left(V_{\alpha}, V_{\delta}\right)$} & \multicolumn{2}{|r|}{$t\left(V_{\alpha}, V_{\delta} \mid r\right)$} \\
\hline & WDs & $\rho_{\mathrm{WD}}\left(M_{\odot} \mathrm{pc}^{-3}\right)$ & WDs & $\rho_{\mathrm{WD}}\left(M_{\odot} \mathrm{pc}^{-3}\right)$ & WDs & $\rho_{\mathrm{WD}}\left(M_{\odot} \mathrm{pc}^{-3}\right)$ \\
\hline $99 \%$ & 6 & $(1.3 \pm 0.8) \times 10^{-5}$ & 3 & $(1.2 \pm 0.8) \times 10^{-5}$ & 3 & $(1.2 \pm 0.8) \times 10^{-5}$ \\
\hline $95 \%$ & 14 & $(2.0 \pm 0.9) \times 10^{-5}$ & 5 & $(1.3 \pm 0.8) \times 10^{-5}$ & 6 & $(1.3 \pm 0.8) \times 10^{-5}$ \\
\hline
\end{tabular}

Table 4. Same as Table 2 after adopting a thick disk velocity distribution convolved with the observation errors.

\begin{tabular}{|c|c|c|c|c|c|c|}
\hline \multirow{2}{*}{$\begin{array}{r}\text { Confid. } \\
\text { level }\end{array}$} & \multicolumn{2}{|r|}{$\psi\left(V_{\alpha}, V_{\delta}\right)$} & \multicolumn{2}{|r|}{$h\left(V_{\alpha}, V_{\delta}\right)$} & \multicolumn{2}{|r|}{$t\left(V_{\alpha}, V_{\delta} \mid r\right)$} \\
\hline & WDs & $\rho_{\mathrm{WD}}\left(M_{\odot} \mathrm{pc}^{-3}\right)$ & WDs & $\rho_{\mathrm{WD}}\left(M_{\odot} \mathrm{pc}^{-3}\right)$ & WDs & $\rho_{\mathrm{WD}}\left(M_{\odot} \mathrm{pc}^{-3}\right)$ \\
\hline $99 \%$ & 14 & $(1.2 \pm 0.5) \times 10^{-5}$ & 8 & $(0.7 \pm 0.4) \times 10^{-5}$ & 5 & $(0.6 \pm 0.4) \times 10^{-5}$ \\
\hline $95 \%$ & 18 & $(1.7 \pm 0.6) \times 10^{-5}$ & 14 & $(1.2 \pm 0.5) \times 10^{-5}$ & 9 & $(1.0 \pm 0.5) \times 10^{-5}$ \\
\hline
\end{tabular}

conservative estimation can be given by selecting only those objects that are not consistent with the "observed" kinematic distribution that results from convolving the projected kinematic distribution of the thick disk (Eq. (3)) with a bivariate Gaussian error distribution with null mean and dispersions, $\left(\epsilon_{V \alpha}, \epsilon_{V \delta}\right)_{(i)}$, corresponding to the velocity errors of the $i$-th object. The velocity errors have been derived by assuming the proper motion errors, $\sigma_{\mu}$, listed in Table 1 of OHDHS, and a more realistic photometric parallax error, $\sigma_{d} / d$, of $25 \%$ (instead of $20 \%$ ).

The different halo WD densities estimated from the objects which are not consistent (at the 95\% and 99\% confidence level) with the new distributions are reported in Tables 3 and 4. Because of the larger velocity thresholds, the number of selected halo WDs is smaller than those reported in Tables 1 and 2. The estimated WD densities, uncorrected for the loss of halo WDs with disk kinematics, decrease proportionally, but are still consistent with $\rho_{\mathrm{WD}} \sim 10^{-5} M_{\odot} \mathrm{pc}^{-3}$. Note that the minimum values, which are reported in Table 4 , have been derived from the data of Salim et al. (2004) who provided distances (and thus volumes) systematically larger than Oppenheimer et al. (2001).

\subsection{On the thick disk model}

As mentioned in Sect. 3.3, our selection criterion also depends implicitly on the choice of the kinematic parameters adopted for the thick disk, whose spatial and kinematical properties are still matter of debate and investigation. Here, we have used the velocity ellipsoid recently derived by Soubiran et al. (2003) from a sample of $\sim 400$ giants with 3D kinematics at a distance of 200-800 pc towards the North Galactic Cap. Their results are very close to the kinematic parameters estimated ${ }^{4}$ by Casertano et al. (1990) and are consistent with various other determinations of the thick disk kinematics, which support velocity dispersions of $40-60 \mathrm{~km} \mathrm{~s}^{-1}$ and an asymmetric drift in the range $30-50 \mathrm{~km} \mathrm{~s}^{-1}$.

\footnotetext{
${ }^{4}$ Casertano et al. (1990) derived $\left(\sigma_{U}, \sigma_{V}, \sigma_{W}, V_{0}\right) \simeq(66,37,38$, $-40) \pm 10 \mathrm{~km} \mathrm{~s}^{-1}$ from a maximum likelihood analysis of high proper motion stars within $500 \mathrm{pc}$ of the Sun.
}

Although controversial, some authors claim the presence of a vertical velocity gradient that supports a thick disk which rotates faster close to the galactic plane (i.e. where the WD sample is localized), than at higher distances, where the studies of the thick disk kinematics have been usually carried out. In particular, Chiba \& Beers (2000), who analyzed 1203 metal poor stars non-kinematically selected, found a rapidly rotating thick disk close to the galactic plane with a small asymmetric drift $V_{0} \simeq-20 \mathrm{~km} \mathrm{~s}^{-1}$ and with velocity dispersions $\left(\sigma_{U}, \sigma_{V}, \sigma_{W}\right) \simeq$ $(46 \pm 4,50 \pm 4,35 \pm 3) \mathrm{km} \mathrm{s}^{-1}$. Moreover, they determined a velocity gradient $\partial V_{0} / \partial|Z| \simeq-30 \pm 3 \mathrm{~km} \mathrm{~s}^{-1} \mathrm{kpc}^{-1}$, that, however, other studies (e.g. Soubiran et al. 2003) do not detect. Nevertheless, a fast rotating thick disk at $Z \approx 0$ was determined ${ }^{5}$ also by Upgren et al. (1997) from a sample of K-M dwarfs in the solar neighborhood ( $d \lesssim 50 \mathrm{pc}$ ) with trigonometric parallaxes and proper motions from the Hipparcos catalogue and radial velocity measurements.

Thus, in order to test the sensitivity of our method with respect to the adopted thick disk model, we repeated the WD selection of the Salim et al. (2004) sample through the distributions $h\left(V_{\alpha}, V_{\delta}\right)$ and $t\left(V_{\alpha}, V_{\delta} \mid r\right)$ derived using the velocity ellipsoid from Chiba \& Beers (2000). The new results are consistent (within $1 \sigma$ ) with the values obtained with the kinematics from Soubiran et al. (2003), although the resulting densities appear typically larger than the previous ones.

For instance, with a $99 \%$ confidence level we find $\rho_{\mathrm{WD}} \simeq$ $(1.7 \pm 0.6) \times 10^{-5} M_{\odot} \mathrm{pc}^{-3}$ for both $h\left(V_{\alpha}, V_{\delta}\right)$ and $t\left(V_{\alpha}, V_{\delta} \mid r\right)$ when the velocity errors are not taken into account (cf. Table 2), while the distributions convolved with the velocity errors provide $\rho_{\mathrm{WD}} \simeq(0.9 \pm 0.5) \times 10^{-5} M_{\odot} \mathrm{pc}^{-3}$ (cf. Table 4). The $95 \%$ confidence level also provides similar but systematically higher new densities up to $(1.8 \pm 0.7) \times 10^{-5} M_{\odot} \mathrm{pc}^{-3}$ and $(3.0 \pm 0.9) \times 10^{-5} M_{\odot} \mathrm{pc}^{-3}$ respectively when the velocity errors are, or are not, convolved with the tangential velocity distributions.

It appears that, with the adopted confidence levels, significantly higher density (e.g. close to $\sim 10^{-4} M_{\odot} \mathrm{pc}^{-3}$ ) may be attained only with disk ellipsoids kinematically much "cooler"

\footnotetext{
5 They estimated a rotation lag of $V_{0} \simeq-28.3 \pm 3.8 \mathrm{~km} \mathrm{~s}^{-1}$ for the "old" disk component with dispersions $\left(\sigma_{U}, \sigma_{V}, \sigma_{W}\right) \simeq(56.1 \pm$ $3.9,34.2 \pm 2.5,31.2 \pm 2.5) \mathrm{km} \mathrm{s}^{-1}$.
} 

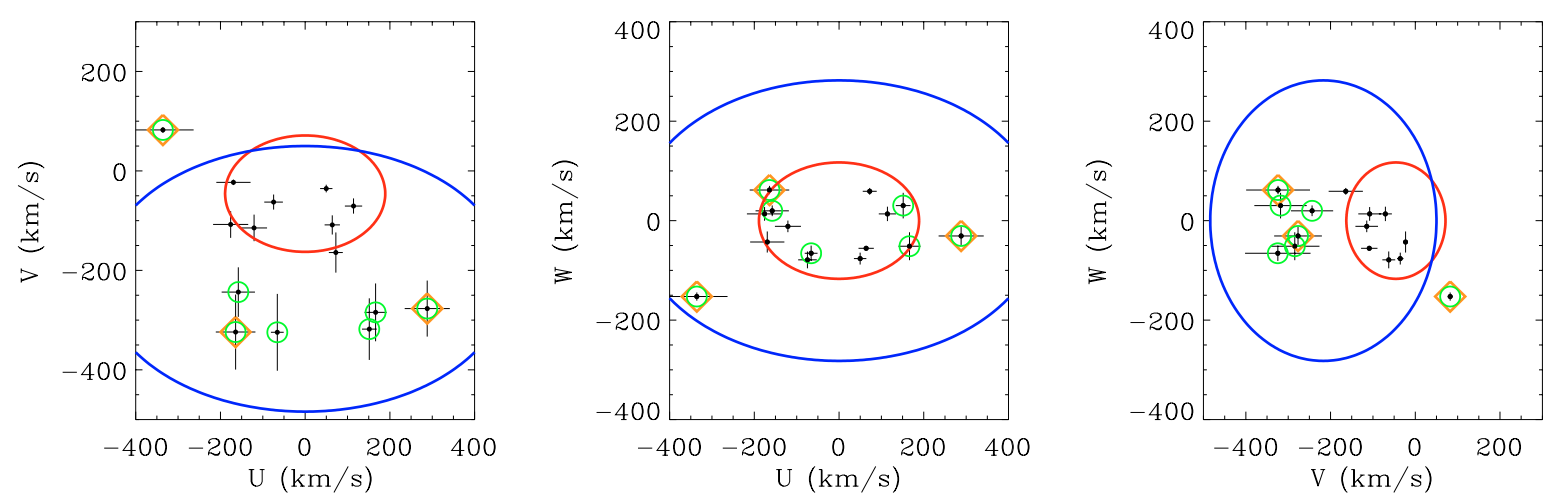

Fig. 4. Velocity distribution $(U, V, W)$ of the subsample of 15 stars with available radial velocity (dots with $1 \sigma$ error bars) from Salim et al. (2004). The objects selected by means of the distributions $h\left(V_{\alpha}, V_{\delta}\right)$ and $t\left(V_{\alpha}, V_{\delta} \mid r\right)$ with a $95 \%$ confidence level (Table 4) are marked with circle and diamond symbols, respectively. The ellipses show the $3 \sigma$ iso-probability contours of the thick disk and halo velocity distribution.

than those expected for a typical thick disk population. For instance, a total density $(8.8 \pm 0.2) \times 10^{-5} M_{\odot} \mathrm{pc}^{-3}$ is only obtained counting all the 41 WDs which are not consistent with the thin disk $^{6}$ kinematics (using $t\left(V_{\alpha}, V_{\delta} \mid r\right)$ with a $95 \%$ confidence level), i.e. summing both halo and thick disk WDs.

\subsection{UVW distribution}

Salim et al. (2004) provide radial velocities for 15 DA WDs, 13 of which were derived from new measurements of the OHDHS sample and two from Pauli et al. (2003), so that, in principle, a more accurate kinematic membership for these objects may be inferred using the information from the full 3D velocities. This requires 3D velocity distributions for kinematically selected samples which are beyond the scope of the current study. However, the availability of both tangential and radial velocities for this subsample offers the possibility to check a posteriori the efficiency of the 2D kinematic analysis adopted in this work and described in Sect. 3 .

To this regard, Fig. 4 shows the $(U, V, W)$ velocities derived from Eq. (2) for the 15 stars with available radial velocities. Those which have been selected with a $95 \%$ confidence level by means of the distributions $h\left(V_{\alpha}, V_{\delta}\right)$ and $t\left(V_{\alpha}, V_{\delta} \mid r\right)$ convolved with the velocity errors (Table 4) are marked with square and diamond symbols. In addition, the $3 \sigma$ iso-probability ellipses of the thick disk and halo velocity distributions, based on the kinematic parameters respectively from Soubiran et al. (2003) and Casertano et al. (1990), are also plotted. The three panels of Fig. 2 indicate that all the likely halo WDs have been properly identified by our kinematic analysis based on the $2 \mathrm{D}\left(V_{\alpha}, V_{\delta}\right)$ distributions, thus supporting the reliability of our selection procedure.

\section{Conclusions}

A kinematically selected sample made of 98 WDs with $\mu>$ $0.33^{\prime \prime} \mathrm{yr}^{-1}$ was published by OHDHS who performed a high proper motion survey over $4165 \mathrm{deg}^{2}$ toward the SGP down to $R 59 F \simeq 19.8$. These data stimulated a number of studies

\footnotetext{
${ }^{6}$ We adopted $\left(\sigma_{U}, \sigma_{V}, \sigma_{W}, V_{0}\right) \simeq(34,21,18,-6) \mathrm{km} \mathrm{s}^{-1}$ from Table 10.4 of Binney \& Merrifield (1998).
}

addressing the issue that a significant part of the dark halo of the Milky Way could be composed of matter in the form of ancient cool WDs. The basic problem - as addressed by several authors - is the criterion to disentangle the mixture of (thick) disk and halo objects on the basis of their kinematic properties and ages.

To this regard, we have implemented a general method for the kinematic analysis of high proper motion surveys and applied it to the identification of reliable halo stars. The kinematically-selected tangential velocity distributions are derived for each star, so that no assumption on the unknown third velocity component, $V_{r}$, nor any approximation on the galactic components $(U, V, W)$, is necessary.

We selected as bona fide halo WDs only those stars whose tangential velocity is inconsistent, at the $95 \%$ and $99 \%$ confidence levels, with the appropriate projected distribution, $h\left(V_{\alpha}, V_{\delta}\right)$ or $t\left(V_{\alpha}, V_{\delta} \mid r\right)$, of the observed thick disk population, thus assuring limited contamination of thick disk objects. Finally, the effect of large velocity errors, which derive from the intrinsic uncertainty of the WD photometric parallaxes, was also discussed and taken into account.

We applied this methodology to the OHDHS sample and selected 10 probable halo WDs (that became 3 after the inclusion of the velocity errors) at a 99\% confidence level. Through the $1 / \mathrm{V}_{\mathrm{Max}}$ method, we estimated a local WD density of $\rho_{\mathrm{WD}} \simeq$ $1 \div 2 \times 10^{-5} M_{\odot} \mathrm{pc}^{-3}$ (i.e. $0.1-0.2 \%$ of the local dark matter) which is consistent with the values found by Gould et al. (1998), as well as by other authors who reanalyzed the OHDHS sample (e.g. Reid et al. 2001; Reylé et al. 2001; Torres et al. 2002; Flynn et al. 2003). The same methodology applied to the OHDHS sample revised by Salim et al. (2004) yields a similar value. These results agree with those found by Carollo et al. (2004) from a first analysis of new data of an independent high proper motion survey in the Northern hemisphere based on material and procedures used for the construction of the GSC-II.

Although affected by a large uncertainty due to the small statistics and low accuracy of the photometric parallaxes, our results clearly indicate that ancient cool WDs do not contribute significantly to the baryonic fraction of the galactic dark halo, as possibly suggested by the microlensing experiments which claimed that $\sim 20 \%$ of the dark matter is formed by compact objects of $\sim 0.5 M_{\odot}$ (Alcock et al. 2000). 
Acknowledgements. We wish to acknowledge the useful discussions with R. Drimmel, S. T. Hodgkin, B. McLean, R. Smart, and L. Terranegra. We also thank the anonymous referee for his/her valuable comments.

Partial financial support for this research came from the Italian Ministry of Research (MIUR) through the COFIN-2001 program.

\section{References}

Alcock, C., Allsman, R. A., Alves, D. R., et al. 2000, ApJ, 542, 281 Bergeron, P., Ruiz, M. T., \& Leggett, S. K. 1997, ApJS, 108, 339 Bergeron, P., Leggett, S. K., \& Ruiz, M. T. 2001, ApJS, 133, 413 Bergeron, P. 2003, ApJ, 586, 201

Binney, J., \& Merrifield, M. 1998, Galactic Astronomy (Princeton Univ. Press)

Carollo, D., et al. 2004, XXVth IAU General Assembly, JD 5, Sidney (Australia), Jul. 16-17, ed. H. Shipman, \& E. M. Sion, in press

Casertano, S., Ratnatunga, K. U., \& Bahcall, J. N. 1990, ApJ, 357, 435

Chiba, M., \& Beers, T. C. 2000, ApJ, 119, 2843

Dehnen, W., \& Binney, J. J. 1998, MNRAS, 298, 387

Flynn, C., Holopainen, J., \& Holmberg, J. 2003, MNRAS, 339, 817

Gilmore, G., Wyse, R. F. G., \& Norris, J. E. 2002, ApJ, 574, L39

Gould, A., Flynn, C., \& Bahcall, J. N. 1998, ApJ, 503, 798
Hambly, N. C., MacGillivray, H. T., Read, M. A., et al. 2001, MNRAS, 326,1279

Hansen, B. M. S. 2001, ApJ, 558, L39

Hansen, B. M. S., \& Liebert, J. 2003, ARA\&A, 41, 465

Koopmans, L. V. E., \& Blandford, R. D. 2001, MNRAS, submitted [arXiv: astro-ph/0107358]

Nelson, C. A., Cook, K. H., Axelrod, T. S., Mould, J. R., \& Alcock, C. 2002, ApJ, 573, 644

Oppenheimer, B. R., Hambly, N. C., Digby, A. P., Hodgkin, S. T., \& Saumon, D. 2001, Science, 292, 698 (OHDHS)

Pauli, E. M., Napiwotzki, R., Altmann, M., et al. 2003, A\&A, 400, 877

Reid, I. N., Sahu, K. C., \& Hawley, S. L. 2001, ApJ, 559, 942

Reylé, C., Robin, A. C., \& Creze, M. 2001, A\&A, L53

Salim, S., Rich, R. Mi., Hansen, B. M., et al. 2004, ApJ, 601, 1075

Schmidt, M. 1975, ApJ, 202, 22

Soubiran, C., Bienaymé, O., \& Siebert, A. 2003, A\&A, 398, 141

Torres, S., García-Berro, E., Burkert, A., \& Isern, J. 2002, MNRAS, 336, 971

Trumpler, R. J., \& Weaver, H. F. 1953, Statistical Astronomy (Univ. of California press)

Upgren, A. R., Ratnatunga, K. U., Casertano, S., \& Weis, E. 1997, Proc. of the ESA Symp. Hipparcos - Venice 97, 13-16 May, 1997, Venice (I), ESA SP-402, 583 